\title{
SOME RESULTS ON DOUBLY STOCHASTIC MATRICES
}

\section{MARVIN MARCUS ${ }^{1}$ AND HENRYK MINC}

1. Introduction. A real $n$-square matrix $S=\left(s_{i j}\right)$ is called doubly stochastic if $s_{i j} \geqq 0$ and

$$
\sum_{j=1}^{n} s_{i j}=\sum_{i=1}^{n} s_{i j}=1, \quad 1 \leqq i, j \leqq n .
$$

Let $J_{n}$ denote the $n$-square doubly stochastic matrix all of whose entries are $1 / n$ and let $\operatorname{per}(S)$ denote the permanent of $S$,

$$
\operatorname{per}(S)=\sum_{\sigma} \prod_{i=1}^{n} s_{i, \sigma(i)}
$$

where the sum is taken over all permutations $\sigma$ of $1, \cdots, n$.

In $1926 \mathrm{~B}$. L. van der Waerden [6] proposed the following problem: What is the minimum of $\operatorname{per}(S)$ as $S$ ranges over all doubly stochastic $n$-square matrices? It is conjectured that

$$
\operatorname{per}(S) \geqq \operatorname{per}\left(J_{n}\right)=\frac{n !}{n^{n}} .
$$

This was proved for $n=3$ in [3] and for all positive semi-definite doubly stochastic matrices in [4]. Clearly (1.1) implies that for any doubly stochastic $S$ there exists a permutation $\sigma$ such that

$$
\prod_{i=1}^{n} s_{i, \sigma(i)} \geqq \frac{1}{n^{n}} .
$$

In the present paper we prove (1.2). This result was obtained independently by A. J. Hoffman, and we are indebted to him for communicating his results to us.

In general the positive semi-definite square root of a positive semidefinite doubly stochastic $A$ need not be a doubly stochastic matrix. For example there exists no doubly stochastic $B$ satisfying $A=B^{2}$ for

$$
A=\left(\begin{array}{ccc}
3 / 4 & 0 & 1 / 4 \\
0 & 3 / 4 & 1 / 4 \\
1 / 4 & 1 / 4 & 1 / 2
\end{array}\right) .
$$

Received by the editors May 8, 1961.

1 The work of this author was supported by U. S. National Science Foundation Grant N.S.F. G-5416. 
The proof of this last statement follows Theorem 2. However, if $a_{i i} \leqq 1 /(n-1), i=1, \cdots, n$, then the positive semi-definite square root of $A$ is doubly stochastic.

A useful tool in investigating combinatorial properties of doubly stochastic matrices is the following form of the Frobenius-König theorem [2]. Let $A$ be any $n$-square matrix and $M$ a subset of the entries of $A$. Each diagonal $d_{\sigma}=\left\{a_{i, \sigma(i)}\right\}, i=1, \cdots, n, \sigma$ a permutation on $1, \cdots, n$, intersects $M$ in at least $k$ entries if and only if $M$ contains an $s \times t$ submatrix of $A$ with $s+t=n+k$.

We use this result to show that if $A$ is $n$-square doubly stochastic and $1 \leqq k \leqq n$, then there are at least $n-k+1$ elements of some diagonal of $A$ which are bounded below by a positive constant depending only on $n$ and $k$ and not on $A$. From this we obtain the following geometric theorem: if $X$ and $Y$ are two orthonormal sets of $n$ vectors in a unitary $n$-space then for each $k$ the vectors $x_{i}$ in $X$ and $y_{i}$ in $Y$ can be so ordered that, for $i=1, \cdots, n-k+1,\left|\left(x_{i}, y_{i}\right)\right|$ is bounded below by a constant $\mu(n, k)$ depending only on $n$ and $k$ and such that $\mu(n, k)>\mu(n, k-1)$. For $k=1$ these results can be found in [5].

Finally we prove that if $(n-1)(n-1) !+1$ of the terms in the permanent expansion of an $n$-square doubly stochastic matrix are equal and nonzero then the matrix must be $J_{n}$.

2. Results.

THEOREM 1. For any doubly stochastic $n$-square matrix $S=\left(s_{i j}\right)$ there exists a permutation $\sigma$ such that

$$
\prod_{i=1}^{n} s_{i, \sigma(i)} \geqq \frac{1}{n^{n}}
$$

with equality if and only if $S=J_{n}$.

Proof. Let

$$
f(t)= \begin{cases}t \log t & \text { if } t>0 \\ 0 & \text { if } t=0\end{cases}
$$

Then $f$ if a strictly convex function on the closed interval $[0,1]$. Define $F$ as a function on $\Omega_{n}$, the convex polyhedron of all $n$-square doubly stochastic matrices, to the reals by

$$
F(S)=\sum_{i, j} f\left(s_{i j}\right)
$$

It is easy to see that $F$ is strictly convex on $\Omega_{n}$ in the sense that $F(\theta S+(1-\theta) T) \leqq \theta F(S)+(1-\theta) F(T), 0<\theta<1$, with equality if and 
only if $S=T$. Observe that, for any permutation matrix $P, F(P S)$ $=F(S)$. Hence if $P$ is a full cycle permutation matrix

$$
\begin{aligned}
F(S) & =\frac{1}{n} \sum_{\alpha=0}^{n-1} F\left(P^{\alpha} S\right) \\
& \geqq F\left(\sum_{\alpha=0}^{n-1} \frac{P^{\alpha}}{n} S\right) \\
& =F\left(J_{n} S\right) \\
& =F\left(J_{n}\right) \\
& =n \log \frac{1}{n},
\end{aligned}
$$

with equality if and only if $P^{\alpha} S=S$ for all $\alpha$, i.e. if and only if $S=J_{n}$. Now, let $S=\left(s_{i j}\right) \in \Omega_{n}$. It is known [1] that there exist permutations $\sigma$ such that $\prod_{i=1}^{n} s_{i, \sigma(i)}>0$. Hence $\max _{\sigma} \prod_{i=1}^{n} s_{i, \sigma(i)}>0$ and we can consider $\max _{\sigma} \sum_{i=1}^{n} \log s_{i, \sigma(i)}$. Let $P=\left(p_{i j}\right)$ be a permutation matrix in $\Omega_{n}$. Clearly

$$
\max _{\sigma} \sum_{i=1}^{n} \log s_{i, \sigma(i)}=\max _{P} \sum_{i, j} p_{i j} \log s_{i j} .
$$

Now, let $T=\left(t_{i j}\right)$ be any matrix in $\Omega_{n}$. Then $\sum_{i, j} t_{i j} \log s_{i j}$ is linear in $T$ and therefore takes its maximum on a permutation matrix, a vertex of the convex polyhedron $\Omega_{n}$. Thus

$$
\begin{aligned}
\max _{P \in \Omega_{n}} \sum_{i, j} p_{i j} \log s_{i j} & =\max _{T \in \Omega_{n}} \sum_{i, j} t_{i j} \log s_{i j} \\
& \geqq \sum_{i, j} f\left(s_{i j}\right) \\
& =F(S) \\
& \geqq n \log \frac{1}{n} .
\end{aligned}
$$

It follows that $\max _{\sigma} \prod_{i=1}^{n} s_{i, \sigma(i)} \geqq 1 / n^{n}$ with equality if and only if $S=J_{n}$. For, if $\max _{\sigma} \prod_{i=1}^{n} s_{i, \sigma(i)}=1 / n^{n}$ then $F(S)=n \log 1 / n$ and thus $S=J_{n}$. Conversely, if $S=J_{n}$ then clearly $\prod_{i=1}^{n} s_{i, \sigma(i)}=1 / n^{n}$ for all $\sigma$.

We next consider conditions under which the unique positive semidefinite determination of the square root of a positive semi-definite matrix is doubly stochastic.

THEOREM 2. Let $A$ be a positive semi-definite $n$-square doubly stochastic matrix with $a_{i i} \leqq 1 /(n-1), i=1, \cdots, n$. Then there exists a doubly stochastic matrix $T$ such that $T^{2}=A$. 
Proof. Let $\alpha_{1}=1, \alpha_{2}, \cdots, \alpha_{n}$ be the characteristic values of $A$. Let $U$ be an orthogonal matrix whose first column entries are all $1 / n^{1 / 2}$ and such that $U^{\prime} A U=\operatorname{diag}\left\{\alpha_{1}, \cdots, \alpha_{n}\right\}=D$. Let

$$
T=U \operatorname{diag}\left\{\left(\alpha_{1}\right)^{1 / 2}, \cdots,\left(\alpha_{n}\right)^{1 / 2}\right\} U^{\prime} .
$$

Then $T^{2}=A$ and we must show that

$$
t_{i j} \geqq 0, \quad \sum_{i} t_{i j}=1, \quad \sum_{j} t_{i j}=1 .
$$

Now $t_{i j}=\sum_{k} u_{i k} \alpha_{k}^{1 / 2} u_{j k}$ so that

$$
\sum_{j} t_{i j}=\sum_{k} u_{i k} \alpha_{k}^{1 / 2} \sum_{j} u_{j k} .
$$

$U$ is orthogonal, hence for $k>1$

$$
\sum_{j} u_{j k}=n^{1 / 2}\left(\sum_{j} u_{j k} \frac{1}{n^{1 / 2}}\right)=0
$$

while $\sum_{j} u_{j \mathrm{r}}=n^{1 / 2}$. Hence

$$
\sum_{j} t_{i j}=u_{i 1} \alpha_{1}^{1 / 2} n^{1 / 2}=\frac{1}{n^{1 / 2}} \cdot 1 \cdot n^{1 / 2}=1 .
$$

Similarly $\sum_{i} t_{i j}=1$. Now suppose $t_{i_{0} j_{0}}<0$ for some $i_{0}, j_{0}$. Then

$$
\sum_{j \neq j_{0}} t_{i_{0} j}=\mu>1 \text { since } \sum_{j} t_{i_{0 j}}=1 .
$$

Then $\mu^{2}=\left(\sum_{j \neq j_{0}} t_{i_{0} j}\right)^{2} \leqq\left(\sum_{j \neq j_{0}} t_{t_{0}}^{2}\right)(n-1)$. Thus

$$
a_{i_{0} i_{0}}=\sum_{j} t_{i_{0 j} j}^{2} \geqq \sum_{j \neq j_{0}} t_{i_{0 j}} \geqq \frac{\mu^{2}}{n-1}>\frac{1}{n-1},
$$

a contradiction.

To see that $a_{i i} \leqq 1 /(n-1)$ cannot be dropped consider the example given in the introduction:

$$
A=\left(\begin{array}{ccc}
3 / 4 & 0 & 1 / 4 \\
0 & 3 / 4 & 1 / 4 \\
1 / 4 & 1 / 4 & 1 / 2
\end{array}\right)
$$

$A$ is positive definite. Now suppose that $A=B^{2}, B=\left(b_{i j}\right) \in \Omega_{3}$. Then

$$
b_{11} b_{12}+b_{12} b_{22}+b_{13} b_{32}=0 \text {, }
$$

$$
b_{21} b_{11}+b_{22} b_{21}+b_{23} b_{31}=0 \text {, }
$$




$$
\begin{aligned}
b_{11}^{2}+b_{12} b_{21}+b_{13} b_{31} & =3 / 4, \\
b_{21} b_{12}+b_{22}^{2}+b_{23} b_{32} & =3 / 4
\end{aligned}
$$

Since all $b_{i j}$ are non-negative, (2.1) implies that the first row or the second column has two 0 entries; the third entry must therefore be 1 . Similarly (2.2) implies that there must be an entry equal to 1 in the second row or in the first column. Since $B$ obviously is not a permutation matrix it can have at most one entry equal to 1 . Thus either $b_{11}$ or $b_{22}$ must be equal to 1 . But (2.3) and (2.4) imply that $b_{11}^{2} \leqq 3 / 4$ and $b_{22}^{2} \leqq 3 / 4$. Contradiction.

THeOREM 3. If $A$ is a doubly stochastic n-square matrix then for each integer $k, 1 \leqq k \leqq n$, there exists a permutation $\sigma$ such that $a_{i, \sigma(i)} \geqq \mu$ for at least $n-k+1$ distinct values of $i$, where

$$
\begin{aligned}
& \mu=\frac{4 k}{(n+k)^{2}} \text { if } n+k \text { is even, } \\
& \mu=\frac{4 k}{(n+k)^{2}-1} \text { if } n+k \text { is odd. }
\end{aligned}
$$

Proof. Suppose that every diagonal of $A$ has fewer than $n-k+1$ elements greater than or equal to $\mu$ on it. That is, in every diagonal there are at least $k$ elements less than $\mu$. Hence by the FrobeniusKönig theorem, $A$ contains an $s \times t$ submatrix $M$ of elements less than $\mu$ where $s+t=n+k$. By permuting rows and columns of $A$ we may assume that $A$ is in the following form:

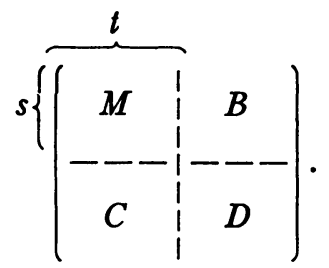

Let $\sum M$ denote the sum of the elements in $M$. Then $\sum M<s t \mu$. Now

$$
\begin{aligned}
& \sum M+\sum B=s \\
& \sum M+\sum C=t ;
\end{aligned}
$$

adding $2 \sum M+\sum B+\sum C=s+t=n+k$. Also $\sum M+\sum B+\sum C$ $+\sum D=\sum A=n$. Hence $\sum M-\sum D=k$ and $\sum M \geqq k$. Also $\sum M$ $<$ st $\mu$ so that 


$$
\mu>\frac{\sum M}{s t} \geqq \frac{k}{\max s t},
$$

where $\max s t$ is the largest value $s t$ takes on, subject to $s+t=n+k$. Now, if $n+k$ is even, then

$$
\max s t=\frac{(n+k)^{2}}{4}
$$

and if $n+k$ is odd, then

$$
\max s t=\left(\frac{n+k-1}{2}\right)\left(\frac{n+k+1}{2}\right)=\frac{(n+k)^{2}-1}{4} .
$$

Hence if $n+k$ is even

$$
\mu>\frac{4 k}{(n+k)^{2}}
$$

and if $n+k$ is odd

$$
\mu>\frac{4 k}{(n+k)^{2}-1}
$$

an impossibility.

The bound $\mu$ in Theorem 3 is best possible in the sense that there exist matrices for each $n$ and $k$ which have the property that no diagonal contains $n-k+1$ elements greater than $\mu$.

Proof. Case (i): $n+k$ is even $\left(\mu=4 k /(n+k)^{2}\right)$. Let

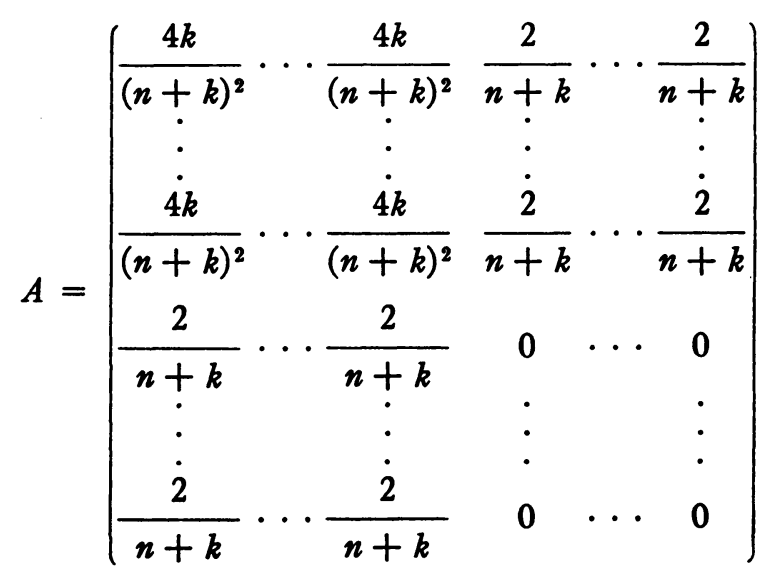

where the upper left submatrix of elements $4 k /(n+k)^{2}$ is $(n+k) / 2$ - 
square. Since the upper left submatrix has the sum of its dimensions equal to $n+k$ it follows that every diagonal has at least $k$ elements equal to $4 k /(n+k)^{2}=\mu$ and hence no diagonal can have $n-k+1$ elements greater than $\mu$.

Case (ii): $n+k$ is odd $\left(\mu=4 k /(n+k)^{2}-1\right)$. Let

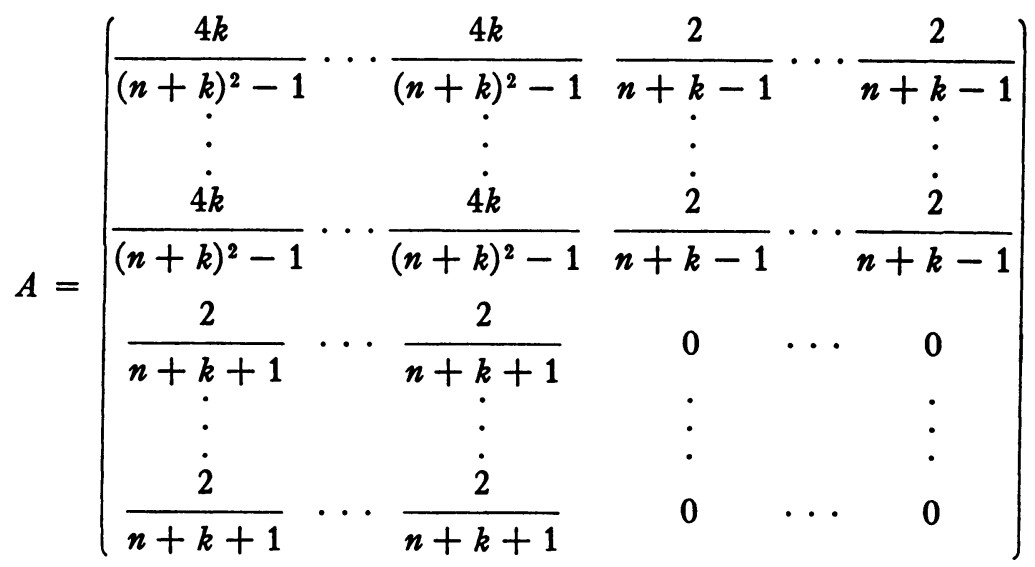

where the upper left corner of elements $4 k /\left((n+k)^{2}-1\right)$ is $(n+k-1) / 2 \times(n+k+1) / 2$.

CoRollary. Let $X$ and $Y$ be two orthonormal sets of $n$ vectors in a unitary n-space. Then for each integer $k$ the vectors $x_{i}$ in $X$ and $y_{i}$ in $Y$ can be ordered so that, for $i=1, \cdots, n-k+1$,

$$
\left|\left(x_{i}, y_{i}\right)\right|^{2} \geqq \begin{cases}\frac{4 k}{(n+k)^{2}} & \text { if } n+k \text { is even, } \\ \frac{4 k}{(n+k)^{2}-1} & \text { if } n+k \text { is odd. }\end{cases}
$$

Proof. Let $a_{i j}=\left|\left(x_{i}, y_{j}\right)\right|^{2}$. Then

$$
1=\left\|x_{i}\right\|^{2}=\sum_{j=1}^{n}\left|\left(x_{i}, y_{j}\right)\right|^{2}
$$

and $1=\left\|y_{j}\right\|^{2}=\sum_{i=1}^{n}\left|\left(x_{i}, y_{j}\right)\right|^{2}$, by Parseval's formula. Hence $A=\left(a_{i j}\right)$ is doubly stochastic and the result follows.

Leмма. If $(n-1)(n-1) !+1$ terms in the permanent of an $n$-square matrix $A$ have a common nonzero value then $A$ has rank 1.

Proof. Note that $A$ cannot have a zero entry and use induction on $n$. 
The lemma is trivial for $n=2$. Assume that if products of entries in $(n-2)(n-2) !+1$ diagonals of an $(n-1)$-square matrix have equal nonzero value then the matrix is of rank 1 .

Let $A$ be any $n$-square matrix which has at least $(n-1)(n-1) !+1$ diagonals with equal products. Then at least $\{((n-1)(n-1) !+1) / n\}$ of these have a common element in the first row, where $\{p\}$ denotes the least integer such that $p \leqq\{p\}$; let the element be $a_{11}$. Now $\{((n-1)(n-1) !+1) / n\} \geqq(n-2)(n-2) !+1$. For

$$
\begin{aligned}
\{((n-1)(n-1) !+1) / n\}-(n-2)(n-2) !-1 \\
\quad=\left\{(n-2) !\left((n-1)^{2}-n(n-2)\right) / n-1+1 / n\right\} \\
\quad=\{((n-2) !+1) / n\}-1
\end{aligned}
$$

which is non-negative for $n \geqq 2$. Hence by the induction hypothesis $A_{11}$ is of rank 1 , where $A_{i j}$ is the submatrix obtained by deleting row $i$ and column $j$ of $A$.

There must be another element $a_{18}$ in the first row of $A$ through which pass at least $\{((n-1)(n-1) !+1-(n-1) !) /(n-1)\}$ of the diagonals which have equal products. But

$$
\begin{aligned}
\{((n-1)(n-1) !+1-(n-1) !) /(n-1)\} \\
\quad=\{(n-1) !-(n-2) !+1 /(n-1)\} \\
\quad=(n-1) !-(n-2) !+1 \\
\quad=(n-2)(n-2) !+1 .
\end{aligned}
$$

Therefore, by the induction hypothesis, $A_{18}$ is also of rank 1 and thus the $(n-1) \times n$ submatrix of $A$ consisting of all rows of $A$ except the first is of rank 1 .

Now we apply the same argument to two elements in the last row and show that the submatrix of $A$ consisting of all rows of $A$ except the last is of rank 1 . Hence $A$ is of rank 1 .

THEOREM 4. If $A$ is an $n$-square doubly stochastic matrix and $A \neq J_{n}$ then at most $(n-1)(n-1) !$ terms in the permanent expansion of $A$ can have a common nonzero value.

Proof. By the above lemma if $\operatorname{per}(A)$ has more than $(n-1)(n-1)$ ! terms with a common nonzero value then $A$ is of rank 1 and it is easily seen that $J_{n}$ is the only rank 1 doubly stochastic matrix.

\section{REFERENCES}

1. G. Birkhoff, Tres observaciones sobre el algebra lineal, Univ. Nac. Tucumán. Rev. Ser. A 5 (1946), 147-151.

2. D. König, Theorie der Graphen, Chelsea, New York, 1950. 
3. M. Marcus and M. Newman, On the minimum of the permanent of a doubly stochastic matrix, Duke Math. J. 26 (1959), 61-72.

4. - The permanent function as an inner product, Bull. Amer. Math. Soc. 67 (1961), 223-224.

5. M. Marcus and R. Ree, Diagonals of doubly stochastic matrices, Quart. J. Math. Oxford Ser. (2) 10 (1959), 296-302. 117.

6. B. L. van der Waerden, Aufgabe 45, Jber. Deutsch. Math. Verein. 35 (1926),

University of British Columbia and

UNIVERSITY OF FLORIDA

\title{
CARDINALITY OF LEVEL SETS OF RADEMACHER SERIES WHOSE COEFFICIENTS FORM A GEOMETRIC PROGRESSION $^{1}$
}

\author{
W. A. BEYER
}

1. Introduction. With $0<r<1$, put

$$
\beta^{*}(\alpha, r)=\left\{x \mid \sum_{i=1}^{\infty} r^{i} R_{i}(x)=\alpha ; 0<x \leqq 1\right\}
$$

where $R_{i}(x)$ is the $i$ th Rademacher function and $|\alpha|<\sum_{i=1}^{\infty} r^{i}$. This paper discusses the cardinality of the set $\beta^{*}(\alpha, r)$ [hereafter denoted by card $\left.\beta^{*}(\alpha, r)\right]$. The only previous discussion known to the author is a remark of Lévy [4]. Denote $(\sqrt{5}-1) / 2$ by $\delta$. In [2] we have shown that if $1>r>\delta$, then the Hausdorff dimension of $\beta^{*}$ is $\geqq 1 / n$ where $n$ is the least $n_{0}$ such that

$$
n_{0}>\left\{\log (2 r-1)-\log \left(r^{2}+r-1\right)\right\} /(-\log r) \text {. }
$$

Note that as $r \rightarrow \delta+, n \rightarrow \infty$. Hence card $\beta^{*}(\alpha, r)=c$ (cardinal number of the continuum) for $1>r>\delta$. It is known that card $\beta^{*}(\alpha, r) \leqq 1$ for $0<r<1 / 2 ; \beta^{*}(\alpha, r)=1$ or 2 for $r=1 / 2$. This leaves the range $1 / 2<r$ $\leqq \delta$ in question. The question is completely settled for $r=\delta$ by Theorem 1 . The range $1 / 2<r<\delta$ is discussed briefly in $\$ 4$.

Presented to the Society, January 22, 1959 under the title Rademacher series with geometric coefficients; received by the editors April 4, 1961.

1 The work reported in this document was performed by Lincoln Laboratory, a center for research operated by Massachusetts Institute of Technology with the joint support of the U. S. Army, Navy, and Air Force under Air Force Contract AF 19(604)5200. 